\title{
BMJ Open Valuation study for a preference-based quality of life measure for dental caries (Dental Caries Utility Index - DCUI) among Australian adolescents - study protocol
}

\author{
Ruvini Hettiarachchi (D) ,1,2 Sanjeewa Kularatna (D) , Joshua Byrnes, ${ }^{1,2}$ \\ Brendan Mulhern, ${ }^{4}$ Gang Chen, ${ }^{5}$ Paul A Scuffham ${ }^{1,2}$
}

To cite: Hettiarachchi R, Kularatna S, Byrnes J, et al. Valuation study for a preferencebased quality of life measure for dental caries (Dental Caries Utility Index - DCUI) among Australian adolescents - study protocol. BMJ Open 2020;10:e038626. doi:10.1136/ bmjopen-2020-038626

- Prepublication history for this paper is available online. To view these files, please visit the journal online (http://dx.doi. org/10.1136/bmjopen-2020038626).

Received 18 March 2020

Revised 19 June 2020

Accepted 27 July 2020

\section{Check for updates}

(C) Author(s) (or their employer(s)) 2020. Re-use permitted under CC BY-NC. No commercial re-use. See rights and permissions. Published by BMJ.

For numbered affiliations see end of article.

\section{Correspondence to} Dr Ruvini Hettiarachchi; ruvini.hettiarachchi@griffithuni. edu.au

\section{ABSTRACT}

Introduction A new health state classification system has been developed for dental caries - Dental Caries Utility Index (DCUI) to facilitate the assessment of oral health interventions in the cost-utility analysis (CUA). This paper reports the protocol for a valuation study, which aims to generate a preference-based algorithm for the classification system for the DCUI.

Methods and analysis Discrete choice experiments (DCEs) will be conducted to value health states generated by the DCUI classification system and preferences for these health states will be modelled to develop a utility algorithm. DCEs produce utility values on a latent scale and these values will be anchored into the full healthdead scale to calculate the quality-adjusted life years in CUA. There is no previous evidence for the most suitable anchoring method for dental caries health state valuation. Hence, we will first conduct pilot studies with two anchoring approaches; DCE including duration attribute and DCE anchoring to worst heath state in Visual Analogue Scale. Based on the pilot studies, the most suitable anchoring method among two approaches will be used in the main valuation survey, which will be conducted as an online survey among a representative sample of 2000 adults from the Australian general population. Participants will be asked to complete a set of DCE choice tasks along with anchoring tasks, basic social-demographic questions, DCUI, a generic preference-based measure and oral health quality of life instrument.

Ethics and dissemination Ethical approval for this study was obtained from the Human Research Ethics Committee Griffith University (reference number HREC/2019/550). The generated algorithm will facilitate the use of the new dental caries preference-based measure in economic evaluations of oral health interventions. The results will be disseminated through journal articles and professional conferences.

\section{INTRODUCTION}

Health economic evaluations play a vital role in assessing healthcare interventions by providing information on which interventions

\section{Strengths and limitations of this study}

- The protocol has been developed for a valuation study, which aims to generate a preference-based utility algorithm for a new health state classification system for dental caries.

- Since there is no previous evidence for the most suitable anchoring method for dental caries health state valuation, pilot studies with two anchoring approaches (DCE including duration attribute and DCE anchoring with Visual Analogue Scale) have been planned prior to the main valuation survey.

- The most suitable valuation approach identified based on the pilot studies will be applied as the main valuation survey to generate the utility algorithm.

- The generated algorithm will facilitate the use of the new dental caries preference-based measure in economic evaluations of dental caries interventions.

- Health states defined by the classification system will be valued by the adult general population sample. The methodological constraints associated with conducting health state valuation studies among the paediatric population, limit the ability to value health states with an adolescents sample.

provides the best value for money. ${ }^{12}$ Costutility analysis (CUA) is a preferred type of health economic evaluation by many Health Technology Assessment authorities across the world. ${ }^{3}$ The CUA compares interventions in terms of their incremental cost per unit of outcome ${ }^{5}$ and quality-adjusted life years (QALYs) is the most common form of outcome measure in CUA. ${ }^{5}$ The QALY incorporates both quantity and quality of life as a single summary outcome measure. The use of QALY as a summary outcome measure allows CUA to compare health interventions across different diseases. ${ }^{5}$

The calculation of QALYs relies on health state utility values (preference weights) 
assigned to the health states in the condition of interest. ${ }^{6}$ Preference-based quality of life measures (PBMs) are used to calculate utility values for QALYs in economic evaluations. PBMs are patient-reported outcome measures that consist of a health state classification system and a set of health state utility values corresponding to each of the health states defined by the classification system. ${ }^{78}$ They are pre-scored, readily available and easy to use rather than the directly eliciting preferences from patients. ${ }^{9}$ There are two types of PBMs. Generic PBMs can be used for any health condition whereas condition-specific PBMs (CSPBMs) are tailored for use among patients with a particular disease or condition of interest. ${ }^{10}$ Generic PBMs such as the EQ-5D ${ }^{11}$ are widely used; however, they may not be sensitive to the changes in some disease conditions since they do not include all relevant dimensions for each disease. ${ }^{10}$ Condition-specific PBMs typically include the dimensions more relevant to a particular disease or condition, thus they may be more responsive to the changes of the disease/condition over time. ${ }^{8}$

Several oral health-related quality of life (OHRQoL) instruments have been developed and validated to date. ${ }^{12}{ }^{13}$ However, none of them are preference-based, thus cannot be used to calculate utility weights for use in health economic evaluations. ${ }^{12}$ Due to the absence of a condition-specific PBM for oral health, researchers are limited to either elicit oral health utility scores via direct valuation methods ${ }^{14}$ and/or use a generic PBM measure ${ }^{15}$ in oral health interventions. ${ }^{16}$ Moreover, children and adolescents are the main focus of publicly funded oral healthcare services in many countries. ${ }^{17}$ They have a different perception about the impact of oral disease on their quality of life compared with adults. Therefore, the availability of paediatric condition-specific PBM for oral health will provide better information on how the disease and the oral health interventions affect the target group children and adolescents ${ }^{18}$ and will facilitate the use of CUA in assessing oral healthcare interventions more effectively. Furthermore, the majority of oral healthcare interventions among children and adolescents focus on dental caries since it is the most common chronic childhood oral disease. ${ }^{1}$ The development of a paediatric condition-specific PBM for dental caries is an important area of research in oral health.

Hence, in the first phase of this study (Hettiarachchi et al, submitted), we developed a classification system for dental caries - Dental Caries Utility Index (DCUI) based on a review of the literature, a series of qualitative interviews with adolescents and with expert opinion; these are accepted methodologies to develop a classification system for a CSPBM. ${ }^{710}$ The DCUI consisted of five items; pain/ discomfort, difficulty eating food/drinking, worried, able to join in activities and appearance, with each item consisted of four levels (table 1). Considering that the mixed dentition period is over by the age of 12 years and those under the age of 12 years have less cognitive ability to

Table 1 Dental Caries Utility Index (DCUI) classification system*

Dimension Description

Pain/discomfort

1. I have no pain or discomfort

2. I have a little pain or discomfort

3. I have quite a lot of pain or discomfort

4. I have lots of pain or discomfort

Difficulty eating food/drinking

1. I have no difficulty in eating food/drinking

2. I have a little difficulty in eating food/drinking

3. I have quite a lot of difficulty in eating food/drinking

4. I have lots of difficulty in eating food/drinking

$\begin{array}{ll}\text { Worried (eg, about losing a tooth, etc) } & \text { 1. I am not worried } \\ & \text { 2. I am a little bit worried } \\ \text { 3. I am quite worried } & \text { 4. I am very worried }\end{array}$

Ability to participate in activities (eg, playing with your friends, sports, schoolwork, etc)

1. I have no difficulty participating in activities

2. I have a little difficulty participating in activities

3. I have quite a lot of difficulty participating in activities

4. I have lots of difficulty participating in activities

$\begin{array}{ll}\text { Appearance } & \text { 1. I am not concerned about my appearance } \\ & \text { 2. I am a little concerned about my appearance } \\ \text { 3. I am quite concerned about my appearance } & \text { 4. I am very concerned about my appearance }\end{array}$

*Hettiarachchi et al, submitted. 
understand the concepts and terms, we included adolescents above 12 years of age during the development of the classification system. Further, the Flesch-Kincaid Reading scores of the finalised instrument was 64.6, indicating that adolescents aged 12 to 13 years can easily understand the classification system. Thus, the target group of this instrument is adolescents above 12 years of age. This paper reports the protocol for a valuation study, which aims to generate a preference-based algorithm for the classification system for the DCUI. To do this, preferences for sets of selected heath states will be elicited that are then modelled to estimate weights for each attribute's level to develop a utility algorithm. The algorithm will facilitate the use of DCUI in health economic evaluation of dental caries interventions among children and adolescents.

\section{METHODS AND ANALYSIS}

\section{Preference elicitation technique}

Different preference elicitation techniques have been adopted to elicit preference weights. The cardinal preference techniques such as standard gamble (SG) and time trade-off (TTO) produce utility values anchored by full health and death. ${ }^{19}$ However, over the recent past, ordinal preference elicitation methods such as discrete choice experiments (DCEs) and ranking orders have become widely used in health state valuations. ${ }^{19}$ A recent systematic review identified 63 health state valuation studies using DCEs and of them, 36 were published during 2016 to $2018 .{ }^{20}$ In a DCE study, participants are requested to state their preference for the series of choices between two or more alternative scenarios describing health problems. ${ }^{21}$ Best-worst scaling (BWS) is a ranking approach, in which participants are asked to state the best and worst from typically three or more sets of items or profiles. ${ }^{22}$ Compared with the traditional valuation methods such as SG and TTO, ${ }^{19}$ DCEs and BWS are typically conducted without an interviewer and compatible with the online surveys that expedites the data collection process. ${ }^{23}$ BWS provides additional information related to worst preferences compared with DCEs; however, previous studies demonstrated that DCEs performed better compared with BWS in health state valuations. ${ }^{24}$ Ordinal preference elicitation methods such as DCE and BWS produce utility values in a latent scale, thus it is important to anchor the utilities generated from these onto the full health to dead scale to calculate the QALY. ${ }^{25}$ Thus, health state valuations studies using DCE or BWS need to include anchoring tasks. In DCE approach, options such as DCE with duration $\left(\mathrm{DCE}_{\mathrm{TTO}}\right)$ are available that can be used as a standalone valuation approach to test anchoring within the task. Considering all these facts, DCE approach will be used as the preference elicitation technique for the present study.

\section{Anchoring methods}

Different methods such as including duration as an additional attribute $\left(\mathrm{DCE}_{\mathrm{TTO}}\right),{ }^{23}$ mapping DCE into TTO, using hybrid models for DCE and TTO, anchoring the worst state using $\mathrm{TTO},{ }^{25}$ including immediate death in pairwise comparisons ${ }^{25}$ and anchoring with Visual Analogue Scale (VAS) have been considered for anchoring DCE values onto the full health to dead scale. The anchoring approaches that need data from separate TTO interviews among participants requires greater time and resources. Dental caries is generally not a lifethreatening condition or associated with serious disabilities. Therefore, pairwise comparisons with death would also not be appropriate since dental caries health state would be predominantly the dominant choice over death or living in full dental health for a considerably shorter duration. DCE utility values obtained from DCE to QALY scale while minimising the drawbacks with conventional TTO. The DCE $_{\text {TTO }}$ method has been used for valuation studies ${ }^{26} 27$ and the methodology has been tested widely. ${ }^{27} 28$ Therefore, the $\mathrm{DCE}_{\text {тто }}$ approach will be used as an anchoring approach for the valuation of DCUI. The VAS has been used in recent health state valuation studies ${ }^{29}$ to generate utility value sets. VAS includes a scale of a single line in which the top of the scale indicates the 'best imaginable health' and the bottom of the scale indicates 'the worst imaginable health'. Individuals are asked to place the health state of interest on this scale. ${ }^{9}$ VAS is simple and easy to understand compared with the SG and TTO methods $^{30}$ and does not attach any trade-off between life years. As mentioned earlier, dental caries is neither life-threatening nor associated with serious disabilities under normal circumstances. Therefore, anchoring with VAS would be an option for a disease condition in which participants will be reluctant to sacrifice life years for the quality of life.

Since there is no previous evidence to identify the most suitable anchoring methods for health state valuations in dental caries, it is worth exploring the two possible options; $\mathrm{DCE}_{\text {тто }}$ and anchoring the worst health state with VAS. Therefore, we will first conduct two pilot studies with two DCE designs; DCE $_{\text {тто }}$ (valuation approach 1) and DCE with VAS (valuation approach 2) in order to identify the most suitable method among these two approaches, and this will then be used to generate the utility algorithm in the main valuation survey.

\section{DCE experimental design and construction of choice sets}

The DCUI instrument consisted of five items, each with four levels. Therefore, there are $1024\left(4^{5}\right)$ possible health states for a full factorial design. As it will not be practical to value all possible combinations, a D-efficient design ${ }^{31}$ will be applied to select a subset of these health states while maximising the efficiency of the survey design. Two separate D-efficient designs with the model specification as multinomial model for valuation approach 1 and 2 (to be explained below) will be generated using Ngene software. ${ }^{32}$ As no previous studies valued dental caries health states derived from a classification system to determine the priors, zero priors will be assumed for all variables to 
generate the D-efficient designs. The number of choice tasks used in the previous health state valuations using DCE were ranged from 12 to 3160 and the majority of studies included more than 151 choice tasks. ${ }^{20}$ The number of choice tasks per respondent were ranged from 2 to $108^{20}$ to value health states derived from a classification system. For this study, eight choice sets per respondent are chosen and block design will be used with eight choice sets in 25 blocks to value 200 pairwise health states. The block design will ensure an equal number of respondents per block. In addition to these eight choice tasks per respondent, the DCE survey will be started, with a practice DCE choice task and a dominant choice question at the beginning of each block as an warm up task and to to allow respondents to be familiar with the DCE tasks.

\section{Pilot study - DCETTO (valuation approach 1)}

For the valuation approach 1, DCE choice tasks will include duration as an additional attribute ( $\mathrm{DCE}_{\text {TTO }}$ choice tasks). Previous DCE studies in dental caries confined to the evaluation of treatment preferences or health services ${ }^{2133}$ rather than the valuation of dental caries health states defined by a classification system. Therefore, no previous literature is available to determine the best levels for the duration attribute in the $\mathrm{DCE}_{\mathrm{TTO}}$ for dental caries.

Dental caries is a chronic disease. Progression of dental caries depends on the balance between pathological factors, such as dietary sugars and bacterial count, and protective factors, such as fluoride and good oral hygiene. ${ }^{34}$ Thus, an assumption was made that the oral health status is constant over time and participants will be in the same caries state in the given duration when including the duration attribute to the choice tasks. Survival duration in the $\mathrm{DCE}_{\text {Tто }}$ could be interpreted as an equivalent to 'marginal willingness to trade life years for an improvement in health status'. ${ }^{23}$ Therefore, it was decided to include 6 months, 1 year, 4 years, 7 years and 10 years as duration levels. Six months is included as it is recommended to visit the dentist every 6 months. 1 year, 4 years, 7 years and 10 years are included as these are the commonly used duration levels in conventional TTO tasks to calculate QALY. $^{35}$ An example DCE $_{\text {TTO }}$ choice task is given in table 2 .

\section{Pilot study - DCE with VAS (valuation approach 2)}

The valuation approach 2 will include DCE tasks without the duration attribute. An example of DCE choice task without the duration attribute is given in table 3. In addition to this, VAS tasks will also be included for anchoring. After DCE paired tasks, a VAS task will be included to value the best health state (11111), the worst heath state of the DCUI (44444), a mild health state, a moderate health state, a severe health state and death in a single VAS scale. The two extreme endpoints of the VAS scale indicate 'best imaginable oral health' (score 100) and 'worst imaginable oral health' (score 0 ). An outline of this task is given in figure 1 .

Further, for both pilot studies, questions will be added at the end of the DCE choice tasks to assess the difficulty in understanding the questions and difficulty in completing the tasks on a response scale of 1 to 4 (not difficult at all to extremely difficult). Time taken to complete the whole survey and each task based on the start time and end time will be assessed to check the feasibility and participant burden.

\section{Main valuation survey}

Based on the pilot studies, the more suitable anchoring method among these two approaches will be chosen and it will be used to generate a utility algorithm in the main survey conducted in the next phase of the study. In addition, these pilot studies will be used to assess whether the survey is launching in the way it is designed, to identify any practical issues or to identify changes required for the main valuation survey.

Table 2 An example of a discrete choice experiment task including duration attribute for the valuation approach 1

Please consider that you are living a life with tooth decay in health state A or B for the period of time specified. During the time, your health state would not change and then will die.

Which health state do you think is better (health state A or health state B)?

\begin{tabular}{|c|c|c|}
\hline & Health state A & Health state B \\
\hline Pain/discomfort & I have no pain or discomfort & I have a little pain or discomfort \\
\hline Difficulty eating foods/drinking & $\begin{array}{l}\text { I have a little difficulty in eating food/ } \\
\text { drinking }\end{array}$ & $\begin{array}{l}\text { I have a little difficulty in eating food/ } \\
\text { drinking }\end{array}$ \\
\hline Worried (eg, about losing your tooth, etc) & I am a little bit worried & I am quite worried \\
\hline $\begin{array}{l}\text { Ability to participate in activities (eg, playing } \\
\text { with your friends, sports, schoolwork, etc) }\end{array}$ & $\begin{array}{l}\text { I have no difficulty participating in } \\
\text { activities }\end{array}$ & $\begin{array}{l}\text { I have a little difficulty participating in } \\
\text { activities }\end{array}$ \\
\hline Duration of life & $\begin{array}{l}\text { Stay this health state for } 1 \text { year and } \\
\text { then die }\end{array}$ & $\begin{array}{l}\text { Stay this health state for } 4 \text { years and } \\
\text { then die }\end{array}$ \\
\hline Which health state do you think is better & $\bigcirc$ & $\bigcirc$ \\
\hline
\end{tabular}


Table 3 An example of a discrete choice experiment task for the valuation approach 2

Please consider that you are living a life with tooth decay in health state A or B for a same period of time. During the time, your health state would not change.

Which health state do you think is better (health state A or health state B)?

\begin{tabular}{lll}
\hline Pain/discomfort & Health state A & Health state B \\
Difficulty eating foods/drinking & I have no pain or discomfort & I have a little pain or discomfort \\
Worried (eg, about losing your tooth, etc) & $\begin{array}{l}\text { I have a little difficulty in eating } \\
\text { food/drinking }\end{array}$ & $\begin{array}{l}\text { I have a little difficulty in eating food/ } \\
\text { drinking }\end{array}$ \\
$\begin{array}{lll}\text { Ability to participate in activities (eg, playing with } \\
\text { your friends, sports, schoolwork, etc) }\end{array}$ & $\begin{array}{l}\text { I am a little bit worried } \\
\text { activities }\end{array}$ & I am quite worried \\
Appearance & I am a little concerned about my & I am not concerned about my \\
& appearance & appearance
\end{tabular}

\section{Study sample}

There are arguments that continue to be held among researchers regarding "whose preference should be valued ${ }^{36}$ for health state valuations of paediatric PBMs. Researchers have argued that the preferences of children and adolescents should be sought, since they have different preferences than adults, and it is them who are receiving the care. ${ }^{37} 38$ However, health state valuation among children is associated with methodological constraints, especially with anchoring tasks. ${ }^{36}$ These tasks would not only be a cognitive burden for children but there are also ethical issues associated with presenting 'death' to children. Thus, previous studies conducted to develop adolescent-specific algorithm followed different approaches. Child Health Utility-9D adolescent-specific algorithms $^{39}$ were developed using two-steps; valuation tasks among adolescents and a separate TTO study with young adults group for anchoring. TTO tasks with adolescent-friendly wordings in face-to-face interviews were used to develop adolescent-specific algorithm for Assessment of Quality of Life (AQoL)-6D. ${ }^{40}$ However, it is common for child and adolescent health state values to be based on an adult general population sample or use proxy responses from parents/guardian. ${ }^{3}$ Most of the existing generic paediatric PBM valuation studies conducted with an adult general population ${ }^{26} 3641$ as it is often public money that will be allocated to fund treatment and therefore it is the preferences of the public that matters. The present study is also planned to elicit preferences for health states from an adult general population sample; this is a highly feasible approach for generating health state values using an online sample. ${ }^{36}$

\section{Sample size and recruitment}

The calculation of sample size for DCE studies is often complex $^{42}$ and researchers suggest different formulae based on several factors. ${ }^{43}$ Johnson and Orme ${ }^{44}$ suggest that the sample size $(\mathrm{n})$ required for the main effects model is based on the number of choice tasks ( $t$ ), the number of alternatives (a) and the number of analysis cells (c), which is equal to the largest number of levels for any of the attributes. Further, Johnson and Orme ${ }^{44}$ recommended a sample size of 300 as a rule of thumb for a quantitative study where there is no intention to compare subgroups and a minimum of about 200 per group for studies that plan to compare groups of respondents to be able to detect significant differences. Further, Lancsar and Louviere ${ }^{45}$ stated that 20 respondents per choice set would be sufficient to estimate a reliable model and a sample size of 1000 to 2000 will be able to produce small CIs.

\section{Pilot study}

Two pilot designs will be tested with an online sample of 400 representative adults (200 representative samples for each design) in Australia.

\section{Main valuation survey}

Based on the literature reviews and similar online surveys, ${ }^{39} 42$ our target is to include a sample size of 2000 representative respondents, which will be compatible with the above requirements for the main survey.

\section{Administration of the survey}

A representative sample of the Australian population in relation to gender and geographical area will be recruited from March 2020. Study participants will be recruited from an existing Australian online panel with the help of online research company SurveyEngine (http://trial.surveyengine.com/about.html). Around $86 \%$ of all households in Australia had access to the Internet at home in 2014 to $2015^{46}$ indicating that online study has a good chance of reaching the target population. Potential participants (adults over 18 years old) who have registered in the online survey panel will receive the invitation for the study and interested participants will be provided the link to the survey. They will be guided through the online survey by screen prompts. The first section of the online survey will be the introduction page where participants will be given all the necessary details about the research project and contact details of the investigator if they need further clarification. 


\section{We would like to know how good or bad the given health scenario.}

We have drawn a scale numbered from 0 to 100 .

0 means the worst oral health you can imagine.

100 means the best oral health you can imagine.

Please read the scenarios below and decide how good or bad you think they are.

Then please click on the button for each scenario and drag and drop into the rating scale

below to indicate your score.

Health State A
Living a life with tooth decay
with following features
I have lots of pain or discomfort
I have lots of difficulty in eating
food/ drinking
I am very worried
I have lots of difficulty
participating in activities
I am very concerned about my
appearance

Score ( )

Health State D

Living a life with tooth decay

with following features

I have a little pain or discomfort

I have a little difficulty in eating

food/drinking

I am a little bit worried

I have no difficulty participating

in activities

I am not concerned about my

appearance

Score ( )

Health State B
Living a life with tooth decay
with following features

I have lots of pain or discomfort
I have lots of difficulty in eating
food/drinking
I am very worried
I have quite difficulty
participating in activities
I am not concerned about my
appearance

Score ()

Health State C
Living a life with tooth decay with
following features
I have quite a lot of pain or discomfort
I have quite a lot of difficulty in eating
food/drinking
I am very worried
I have no difficulty participating in
activities
I am not concerned about my
appearance

Score ( )
Participants will be informed that the participation in this survey will be voluntary and the survey data will be anonymous and confidential. At the end of the introduction page, participants will be requested to provide their consent. Once the participant gives their consent, they will move to the next section and continue the rest of the survey. Consenting participants will be guided through the online survey by screen prompts and this will enable them to complete the tasks at their convenience. The next section of the survey will include screening questions (age, sex and which state they live in) followed by DCUI and a set of DCE choice tasks and anchoring tasks. Then the participants will be asked to complete a generic preference-based QoL instrument (the EuroQoL 5D 5L questionnaire - EQ-5D-5L), ${ }^{47}$ an OHRQoL instrument (Oral health impact profile-14 - OHIP-14) ${ }^{48}$ and questions regarding basic social-demographic characteristics, oral health status and frequency of dental visits. EQ-5D-5L and OHIP-14 are generic and OHRQoL instruments commonly used among Australian general population $^{49}$ to assess generic and OHRQoL, respectively. The OHIP-14 has been shown adequate psychometric properties and validated extensively among both adults ${ }^{50}$ and adolescents population. $^{51}$ 
Patient and public involvement

No patient involved.

\section{Analytical plan}

All data will be cleaned prior to the analysis and data will be analysed using Stata V.15.1. ${ }^{52}$ DCE data will be analysed using a conditional logit model under a random utility framework, which assumes that respondents choose the alternative that maximises their utility. ${ }^{53}$ The utility function consists of a vector of observable attributes as well as a random error term. ${ }^{19}$ Both the main effects and the interactions among attributes will be considered.

\section{Pilot study - DCE}

The observable component $\left(\mu_{\mathrm{ij}}\right)$ of the utility function will be estimated using a conditional logit model as in Equation 1 . The life years $t$ will be included as a continuous variable.

$$
U_{i s j}=\alpha t_{i s j}+\beta x_{i j j} . t_{i j j}+\epsilon_{i j j}
$$

$\mathrm{U}_{\mathrm{isj}}=\mathrm{U}$ tility of the option $\mathrm{j}$ in choice set $\mathrm{s}$ for survey respondent i $\mathrm{x}=a$ vector contains five DCUI dimensions while each dimension is estimated using three dummy variables (with 'no problems' served as the reference level within each attribute).

$\mathrm{t}=$ life years

Anchoring results to a health utility scale will be performed based on the methodology described by Bansback et $a l .^{23}$ The objective is to derive the mean utility value of the state $\mathrm{x}_{\mathrm{ij}}$ in DCE that correspond to a 10-year TTO value.

$$
\hat{V}_{j}^{D C E}=1+\frac{\hat{\beta}_{2}}{\hat{\beta}_{2}} x_{j}
$$

$V_{j}=$ value of the health state $h_{\mathrm{j}}$ anchored on the health utility scale

$\hat{\beta}_{2}=$ disutility of living with the health state $\mathrm{h}_{\mathrm{j}}$ for 1 year

$\hat{\beta_{2}}=$ coefficient represents the value of living in full health for 1 year

$x_{j}=$ value for each health state

Based on this formula, the sample mean $\mathrm{DCE}_{\text {тто }}$ for the health state $h_{j}$ can be calculated from the coefficients of the conditional logit model.

\section{Pilot study - DCE with VAS (valuation approach 2)}

To obtain the coefficients in DCE latent scale, data from the DCE choice tasks in valuation approach 2 will be modelled with an appropriate regression model with the following specification. The best-fitted model will be selected based on the statistical significance of coefficients, the amount of explained variance and mean absolute error (absolute difference between the observed value and estimated value in each health state).

$$
U_{i j}=\propto_{i}+\beta_{1} x_{1}+\beta_{2} x_{2}+\beta_{3} x_{3}+\beta_{4} x_{4}+\beta_{5} x_{5}
$$

$\mu_{\mathrm{ij}}=$ observable component of the utility function

$x=$ vector contains five DCUI dimensions while each dimension is estimated using three dummy variables (with 'no problems' served as the reference level within each attribute)

$\beta=$ coefficient for each dimension

Anchoring to the full health-dead scale of the VAS anchoring task will be performed based on the methodology proposed by Brazier et $a l^{30}$ and Rowen $e t a l .{ }^{25}$ Brazier et $a \vec{l}^{30}$ proposed a formula (Equation 4) to convert the health state values in to full health-dead scale based on the value obtained for best health state and death from VAS health state valuation tasks and this has been used in previous studies. ${ }^{29}$ Raw VAS scores for worst heath state of the DCUI (44444), a mild health state, a moderate health state and a severe health state will be converted to full health-dead scale using Equation 4.

$$
V_{h}=\frac{S_{h}-S_{\text {dead }}}{S_{11111}-S_{\text {dead }}}
$$

$\mathrm{V}_{\mathrm{h}}=$ adjusted VAS rating for health state $\mathrm{h}$

$\mathrm{S}_{\mathrm{h}}=$ respondent's unadjusted VAS score for state $\mathrm{h}$

$\mathrm{S}_{\text {dead }}=$ respondent's assigned VAS score for the health state 'death'

$\mathrm{S}_{11111}=$ respondent's assigned VAS score for a state 11111 (best state)

Then the value of the worst state in the DCE model will be anchored based on anchoring with worst health states and with mapping DCE onto VAS. Rowen et $a l^{25}$ converted the coefficients on a latent utility scale estimated in DCE data onto the full health-dead scale using the estimated TTO value of the worst state. The same methodology would be followed here and based on the adopted formula (Equation 5) from Rowen et al, ${ }^{25}$ DCE data will be anchored to the worst state value obtained in VAS.

$$
\beta r \lambda=\beta \lambda * \frac{w V A S}{w D C E}
$$

$\beta \lambda \partial=$ rescaled coefficient for level $\lambda$ of dimension $\partial$ wVAS = estimated VAS value for the worst state generated using equation 4 and 5

wDCE $=$ DCE value for the worst state estimated using the DCE model

For the anchoring with mapping DCE onto VAS, mean VAS values obtained for the worst health state of the DCUI (44444), a mild health state, a moderate health state and a severe health state will be used as in Equation 6 .

$$
\begin{gathered}
d V A S_{j}=1-V A S_{j} \\
d D C E_{j}=1-D C E_{j} \\
d V A S_{j}=f\left(d D C E_{j}\right)+\varepsilon_{j}
\end{gathered}
$$

$\mathrm{VAS}=$ mean VAS value of health state $\mathrm{j}$

$\mathrm{DCE} j=$ modelled latent utility value of health state $j$

$\mathrm{dVASj}=$ disutility of the mean VAS value of health state $\mathrm{j}$

$\mathrm{dDCE} j=$ disutility of the modelled latent utility value of health state $\mathrm{j}$

$$
\varepsilon=\text { error term }
$$

Comparison of valuation approach 1 and 2 to assess the most suitable anchoring method for dental caries health state valuation As these two DCE designs are different, they are not directly comparable. However, the pilot studies aim to 
identify the most suitable method for anchoring among these two approaches. Therefore, data from the two valuation approaches will be compared concerning the respondents' self-reported difficulty of the tasks (based on the answers provided for the two questions - how difficult to understand the questions and how difficult to complete the tasks), dropout rate, time taken by each individual on the whole survey and for each DCE task.

Further anchored coefficients obtained from the valuation approach 1 and 2, as outlined in the analytical plan, will be examined for the sign and order of the coefficients; that is, the sign of the duration coefficient should be positive (since utility increase with the time living in full health) and levels in each domain should follow a logical order in which more severe should have larger utility decrement.

\section{Main valuation survey}

Once the most suitable valuation approach is decided based on the pilot surveys, that approach will continue as the main valuation survey to generate the utility algorithm. For the main survey, participants' characteristics will be assessed and a $\chi^{2}$ test will be used to assess sample representativeness of the Australian general population. The EQ-5D-5L, OHIP-14, DCUI and self-reported oral health variables data will be analysed to assess the respondents' QoL and oral health status. DCE tasks data of the main survey will be modelled based on the selected approach from the pilot study, as outlined in the analytical plan. The utility values generated from the main survey can then be used to calculate QALY in economic evaluation in dental caries interventions.

\section{ETHICS AND DISSEMINATION}

Ethical approval for this study was obtained from the Human Research Ethics Committee, Griffith University (HREC/2019/550). Informed consent will be obtained from the participants prior to starting the survey. Participants are only required to complete the survey; therefore, there is negligible or low risk for the participants. There are no potential adverse events/side effects. The research team will receive only de-identified anonymous data. The data obtained by this research project will be stored securely with a password-protected computer, and secure server in Griffith University until data will be destroyed after the 5 years minimum period of retention in accordance with University policy. Any personal details that will lead to the identification of individual participants will not be included in any report or publication arising from this research project. Dissemination of the study results will be through the publication of manuscripts in academic journals and conference presentations.

\section{Author affiliations}

${ }^{1}$ Centre for Applied Health Economics, School of Medicine, Griffith University, Nathan, Queensland, Australia

${ }^{2}$ Menzies Health Institute Queensland, Griffith University, Nathan, Queensland, Australia
${ }^{3}$ Australian Centre for Health Services Innovation, Queensland University of Technology, Institute of Health and Biomedical Innovation, Kelvin Grove, Queensland, Australia

${ }^{4}$ Centre for Health Economics Research and Evaluation, University of Technology Sydney, Sydney, New South Wales, Australia

${ }^{5}$ Centre for Health Economics, Monash University, Clayton, Victoria, Australia

Twitter Ruvini Hettiarachchi @ruvinihettiara

Contributors RH contributed to designing the study and draft of the article. SK, $\mathrm{JB}, \mathrm{BM}, \mathrm{GC}$ and PS contributed to the design of the study, editing and revising the article. All co-authors read and agreed the final version of the article to be published.

Funding The authors have not declared a specific grant for this research from any funding agency in the public, commercial or not-for-profit sectors.

\section{Competing interests None declared.}

Patient and public involvement Patients and/or the public were not involved in the design, or conduct, or reporting, or dissemination plans of this research.

Patient consent for publication Not required.

Provenance and peer review Not commissioned; externally peer reviewed.

Open access This is an open access article distributed in accordance with the Creative Commons Attribution Non Commercial (CC BY-NC 4.0) license, which permits others to distribute, remix, adapt, build upon this work non-commercially, and license their derivative works on different terms, provided the original work is properly cited, appropriate credit is given, any changes made indicated, and the use is non-commercial. See: http://creativecommons.org/licenses/by-nc/4.0/.

ORCID iDs

Ruvini Hettiarachchi http://orcid.org/0000-0003-3501-2645

Sanjeewa Kularatna http://orcid.org/0000-0001-5650-154X

\section{REFERENCES}

1 Tonmukayakul U, Calache $\mathrm{H}$, Clark R, et al. Systematic review and quality appraisal of economic evaluation publications in dentistry. $J$ Dent Res 2015;94:1348-54.

2 Morgan M, Mariño R, Wright C, et al. Economic evaluation of preventive dental programs: what can they tell us? Community Dent Oral Epidemiol 2012;40:117-21.

3 Thorrington D, Eames K. Measuring health utilities in children and adolescents: a systematic review of the literature. PLoS One 2015;10:e0135672.

4 Canadian Agency for Drugs and Technologies in Health. Guidelines for the economic evaluation of health technologies: Canada. 3rd edn. Ottawa, Canada: Canadian Agency for Drugs and Technologies in Health, 2006.

5 Greenberg D, Pliskin JS. Preference-based outcome measures in cost-utility analyses. A 20 -year overview. Int J Technol Assess Health Care 2002;18:461-6.

6 Torrance GW. Measurement of health state utilities for economic appraisal. J Health Econ 1986;5:1-30.

7 Stevens K, Palfreyman S. The use of qualitative methods in developing the descriptive systems of preference-based measures of health-related quality of life for use in economic evaluation. Value Health 2012;15:991-8.

8 Rowen D, Brazier J, Ara R, et al. The role of Condition-Specific Preference-Based measures in health technology assessment. Pharmacoeconomics 2017;35:33-41.

9 Whitehead SJ, Ali S. Health outcomes in economic evaluation: the QALY and utilities. Br Med Bull 2010;96:5-21.

10 Brazier JE, Rowen D, Mavranezouli I, et al. Developing and testing methods for deriving preference-based measures of health from condition-specific measures (and other patient-based measures of outcome). Health Technol Assess 2012;16.

11 Brooks R. EuroQol: the current state of play. Health Policy 1996;37:53-72.

12 Hettiarachchi RM, Kularatna S, Byrnes J, et al. Pediatric quality of life instruments in oral health research: a systematic review. Value Health 2019;22:129-35.

13 Thomson WM, Broder HL. Oral-Health-Related quality of life in children and adolescents. Pediatr Clin North Am 2018;65:1073-84.

14 Bhuridej P, Kuthy RA, Flach SD, et al. Four-Year cost-utility analyses of sealed and nonsealed first permanent molars in lowa Medicaidenrolled children. J Public Health Dent 2007;67:191-8. 
15 Koh R, Pukallus M, Kularatna S, et al. Relative cost-effectiveness of home visits and telephone contacts in preventing early childhood caries. Community Dent Oral Epidemiol 2015;43:560-8.

16 Hettiarachchi RM, Kularatna S, Downes MJ, et al. The costeffectiveness of oral health interventions: a systematic review of cost-utility analyses. Community Dent Oral Epidemiol 2018;46:118-24.

17 Gherunpong S, Tsakos G, Sheiham A. Developing and evaluating an oral health-related quality of life index for children; the CHILD-OIDP. Community Dent Health 2004;21:161-9.

18 Marshman Z, Robinson PG. Child and adolescent oral health-related quality of life. Semin Orthod 2007;13:88-95.

19 Ali S, Ronaldson S. Ordinal preference elicitation methods in health economics and health services research: using discrete choice experiments and ranking methods. Br Med Bull 2012;103:21-44.

20 Mulhern B, Norman R, Street DJ, et al. One method, many methodological choices: a structured review of Discrete-Choice experiments for health state valuation. Pharmacoeconomics 2019;37:29-43.

21 Lord J, Longworth L, Singh J, et al. Oral health guidance economic analysis of oral health promotion approaches for dental teams. 06/02/2015 ED. Birmingham \& Brunel Consortium External Assessment Centre (BBC EAC), 2015.

22 Cheung KL, Wijnen BFM, Hollin IL, et al. Using Best-Worst scaling to investigate preferences in health care. Pharmacoeconomics 2016;34:1195-209.

23 Bansback N, Brazier J, Tsuchiya A, et al. Using a discrete choice experiment to estimate health state utility values. J Health Econ 2012;31:306-18.

24 Krucien N, Watson V, Ryan M. Is Best-Worst scaling suitable for health state valuation? A comparison with discrete choice experiments. Health Econ 2017;26:e1-16.

25 Rowen D, Brazier J, Van Hout B. A comparison of methods for converting DCE values onto the full health-dead QALY scale. Med Decis Making 2015;35:328-40.

26 Rowen D, Mulhern B, Stevens K, et al. Estimating a Dutch value set for the pediatric Preference-Based CHU9D using a discrete choice experiment with duration. Value Health 2018;21:1234-42.

27 Mulhern B, Bansback N, Hole AR, et al. Using discrete choice experiments with duration to model EQ-5D-5L health state preferences: testing experimental design strategies. Med Decis Making 2016;37:285-97.

28 Bansback N, Hole AR, Mulhern B, et al. Testing a discrete choice experiment including duration to value health states for large descriptive systems: addressing design and sampling issues. Soc Sci Med 2014;114:38-48.

29 Goudarzi R, Zeraati H, Akbari Sari A, et al. Population-Based preference weights for the EQ-5D health states using the visual analogue scale (vas) in Iran. Iran Red Crescent Med J 2016;18:e21584.

30 Brazier J, Green C, McCabe C, et al. Use of visual analog scales in economic evaluation. Expert Rev Pharmacoecon Outcomes Res 2003;3:293-302.

31 Reed Johnson F, Lancsar E, Marshall D, et al. Constructing experimental designs for discrete-choice experiments: report of the ISPOR conjoint analysis experimental design good research practices Task force. Value Health 2013;16:3-13.

32 ChoiceMetrics. Ngene 1.2 User Manual \& reference Guide [program]. Sydney, Australia: ChoiceMetrics, 2018.
33 Barber S, Bekker H, Marti J, et al. Development of a Discrete-Choice experiment (DCE) to elicit adolescent and parent preferences for hypodontia treatment. Patient 2019;12:137-48.

34 Featherstone JDB. Caries prevention and reversal based on the caries balance. Pediatr Dent 2006;28:128-32.

35 Scalone L, Stalmeier PFM, Milani S, et al. Values for health states with different life durations. Eur J Health Econ 2015;16:917-25.

36 Kreimeier S, Greiner W. EQ-5D-Y as a health-related quality of life instrument for children and adolescents: the instrument's characteristics, development, current use, and challenges of developing its value set. Value Health 2019;22:31-7.

37 Montgomery SM, Kusel J. The prevalence of child-specific utilities in NICE appraisals for paediatric indications: rise of the economic orphans? Expert Rev Pharmacoecon Outcomes Res 2016;16:347-50.

38 Ratcliffe J, Huynh E, Stevens K, et al. Nothing about us without us? A comparison of adolescent and adult Health-State values for the child health Utility-9D using profile case Best-Worst scaling. Health Econ 2016;25:486-96.

39 Ratcliffe J, Huynh E, Chen G, et al. Valuing the child health utility 9D: using profile case best worst scaling methods to develop a new adolescent specific scoring algorithm. Soc Sci Med 2016;157:48-59.

40 Moodie M, Richardson J, Rankin B, et al. Predicting time trade-off health state valuations of adolescents in four Pacific countries using the assessment of quality-of-life (AQoL-6D) instrument. Value Health 2010;13:1014-27.

41 Chen G, Ratcliffe J. A review of the development and application of generic Multi-Attribute utility instruments for paediatric populations. Pharmacoeconomics 2015;33:1013-28.

42 Wang B, Chen G, Ratcliffe J, et al. Adolescent values for immunisation programs in Australia: a discrete choice experiment. PLoS One 2017;12:e0181073.

43 de Bekker-Grob EW, Donkers B, Jonker MF, et al. Sample size requirements for Discrete-Choice experiments in healthcare: a practical guide. Patient 2015;8:373-84.

44 Orme B. Sample size issues for conjoint analysis studies. Sawthooth software research paper series. Squim WA, USA: Sawthooth Software Inc, 1998.

45 Lancsar E, Louviere J. Conducting discrete choice experiments to inform healthcare decision making: a user's guide. Pharmacoeconomics 2008;26:661-77.

46 Statistics ABo. 8146.0 - Household Use of Information Technology, Australia, 2014-15. Canbera: Austarlain Bureau of Statistics, 2016.

47 EuroQol Group. EQ-5D-5L about. Available: https://euroqol.org/eq5d-instruments/eq-5d-5l-about/ [Accessed 12 Dec 2019].

48 Slade GD. Derivation and validation of a short-form oral health impact profile. Community Dent Oral Epidemiol 1997;25:284-90.

49 Brennan DS. Oral health impact profile, EuroQol, and assessment of quality of life instruments as quality of life and health-utility measures of oral health. Eur J Oral Sci 2013;121:188-93.

50 van de Rijt LJM, Stoop CC, Weijenberg RAF, et al. The influence of oral health factors on the quality of life in older people: a systematic review. Gerontologist 2020;60:e378-94.

51 Ferrando-Magraner E, García-Sanz V, Bellot-Arcís C, et al. Oral health-related quality of life of adolescents after orthodontic treatment. A systematic review. J Clin Exp Dent 2019;11:e194-202.

52 STATA 15.1 [program]. Texas, USA: StataCorp LP 2015.

53 McFadden D. Conditional logit analysis of qualitative choice behaviour. Analysis of qualitative choice behaviour 1973. 